\title{
Partial pancreatic resection along the embryological fusion plane - no longer a fantasy
}

\author{
M. Łącka, D. Łaski, S. Hać, M. Szymański \\ Department of General, Endocrine and Transplant Surgery, Medical University of Gdansk, Poland \\ [Received: 9 September 2019; Accepted: 5 October 2019]
}

Background: The embryological connection between the dorsal and ventral pancreatic regions divides the pancreas into two segments. This anatomical dependence allows segmental pancreatic resection through the embryological fusion plane (EFP). The advantages of limited pancreatic resection are the preservation of the natural continuity and function of the gastrointestinal tract and the avoidance of the metabolic and endocrine consequences of total resection of the pancreas and the duodenum.

Materials and methods: Two patients are described who underwent anatomical segmentectomy of the pancreatic head along the EFP for the treatment of pancreatic cystic tumour and main duct intraductal papillary-mucinous neoplasm. The authors suggested diagnostic and intraoperative management leading to qualification for pancreatic resection along the EFP.

Results: Pancreas and duodenum sparing surgery is an opportunity for patients in terms of the post-operative quality of life. Indications for this kind of surgery are limited and case selection is very difficult. The procedure for embryological bud resection is highly complicated includes a high rate of possible complications. On the other hand high volume centres may offer this procedure at an acceptable rate of complications in selected cases.

Conclusions: Accurate diagnosis with a vascular anatomy and biliary and pancreatic duct configuration give grounds to analyse pancreas-sparing surgery. The operation plan requires careful three-dimensional planning and an experienced team. Bipolar electrocautery, micro-surgical tools and intraoperative cholangiography and pancreatography are helpful. (Folia Morphol 2020; 79, 3: 640-644)

Key words: intraductal papillary-mucinous neoplasm, pancreas segmentectomy, pancreatic sparing resection

\section{INTRODUCTION}

The pancreas is made up of three compounds one dorsal and two ventral; right and left. The left compound disappears during embryogenesis. The right one approaches the dorsal and grows with it and creates the embryological fusion plane (EFP) $[3,6,8-10,13]$.
The main mass of the pancreas - the upper part of the head, the entire trunk and tail, are formed from the dorsal bud. The lower part of the head is formed from the ventral compound $[3,8]$.

The embryological connection between the dorsal and ventral pancreatic regions divides the pancreas

Address for correspondence: D. Łaski, PhD, Department of General, Endocrine and Transplant Surgery, Medical University of Gdansk, ul. M. Smoluchowskiego 17, 80-214 Gdańsk, Poland, tel: +48 5834930 10, fax: +48 58349 30 20, e-mail: dlaski@gumed.edu.pl 


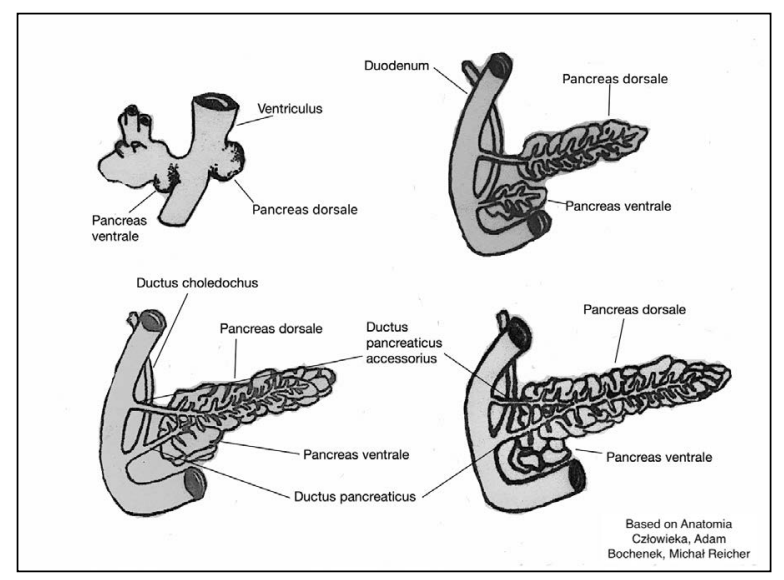

Figure 1. The embryological connection between the dorsal and ventral pancreatic regions divides the pancreas into two segments.

into two segments, making a segmental pancreatic resection through the EFP feasible (Fig. 1) $[8,11]$.

This anatomical dependence allows pancreas and duodenum sparing treatments in cases of benign and low-grade neoplasms of the pancreas $[2,7,14]$. The advantages of limited pancreatic resection are not only the preservation of the natural continuity and function of the gastrointestinal tract, but also the avoidance of the metabolic and endocrine consequences of total resection of the pancreas and duodenum $[4,12,14]$.

\section{CASE REPORTS}

Two patients are described who underwent anatomical segmentectomy of the pancreatic head along the EFP for the treatment of pancreatic cystic tumour and main duct intraductal papillary-mucinous neoplasm (IPMN). The authors suggested diagnostic and intraoperative management leading to qualification for pancreatic resection along the EFP.

\section{Case 1}

A 64-year-old woman was admitted to the Department of General, Endocrine and Transplant Surgery with a long history of abdominal mass. In the computed tomography (CT) scan, the extrahepatic biliary ducts were distended up to $21 \mathrm{~mm}$, intrahepatic ducts were marked up to $3-4 \mathrm{~mm}$, and polycyclic mass $(60 \times 66 \times 93 \mathrm{~mm})$ filled with dense fluid was observed in the pancreatic head. The lesion was modelling the pre-pyloric part of the stomach and the duodenum loop, without the obvious features of infiltration of their walls. The upper mesenteric arteries and coeliac trunk had no radiological traces of invasion. The Wirsung duct in the tail of the pancreas was distended up to $17 \mathrm{~mm}$ (Fig. 2).

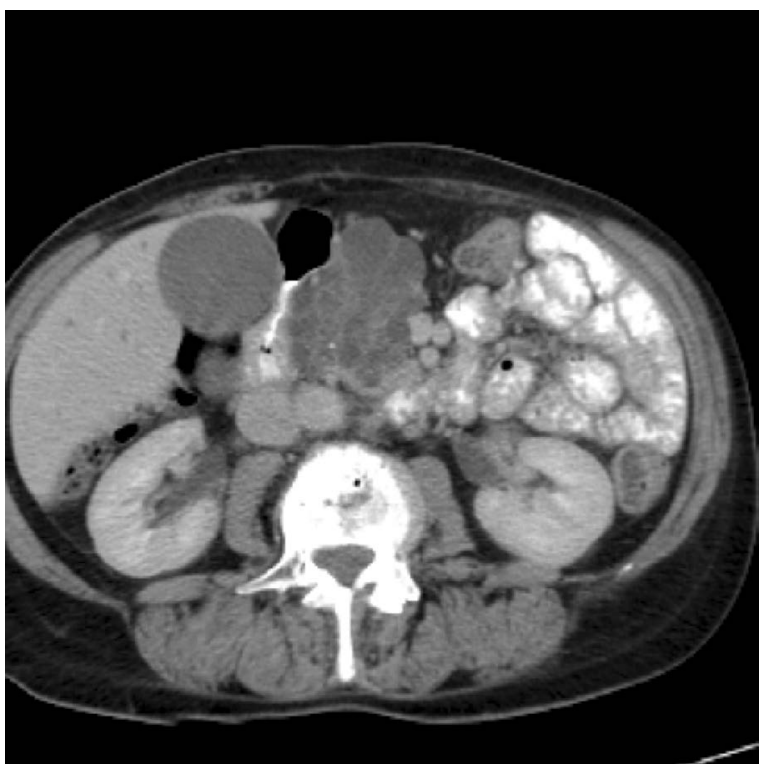

Figure 2. 64-year-old patient computed tomography scan, polycyclic mass $(60 \times 66 \times 93 \mathrm{~mm})$ filled in the pancreatic head.

No radiological signs of malignancy were present. Radiological diagnosis was mucinous cystic neoplasm of the pancreas without malignant transformation with outflow obstruction from the ventral pancreatic duct. The ventral duct was not affected by the lesion and was of normal size.

The patient did not present any other symptoms; the history of pancreatitis was negative. The patient was qualified for surgical treatment.

Surgery. During the procedure, a polycystic tumour in the head of the pancreas was confirmed. The common bile duct was distended to $3 \mathrm{~cm}$, and the gallbladder was distended to $20 \mathrm{~cm}$. A part of the head of the pancreas originating from the dorsal compound was resected through the EFP. An intraoperative histopathological examination of the cut line did not reveal malignant traits. An intraoperative pancreatography through the duct on the cut line of the pancreatic stump was performed. No leakage was found from the pancreatic ducts, and no anomalous pancreatic ducts were found. After the cholecytectomy the T-tube was left in the common bile duct. Cholangiography confirmed the normal outflow to the duodenum and complete decompression of the common bile duct after cystic tumour resection (Figs. 3, 4). The cut line of the pancreas was connected to the small bowel on the Roux loop.

The postoperative period was uneventful. The final postoperative pathology revealed adenoma serosum oligocellulare. Fourteen days after surgery, an outpatient cholangiography was performed and the T-tube 


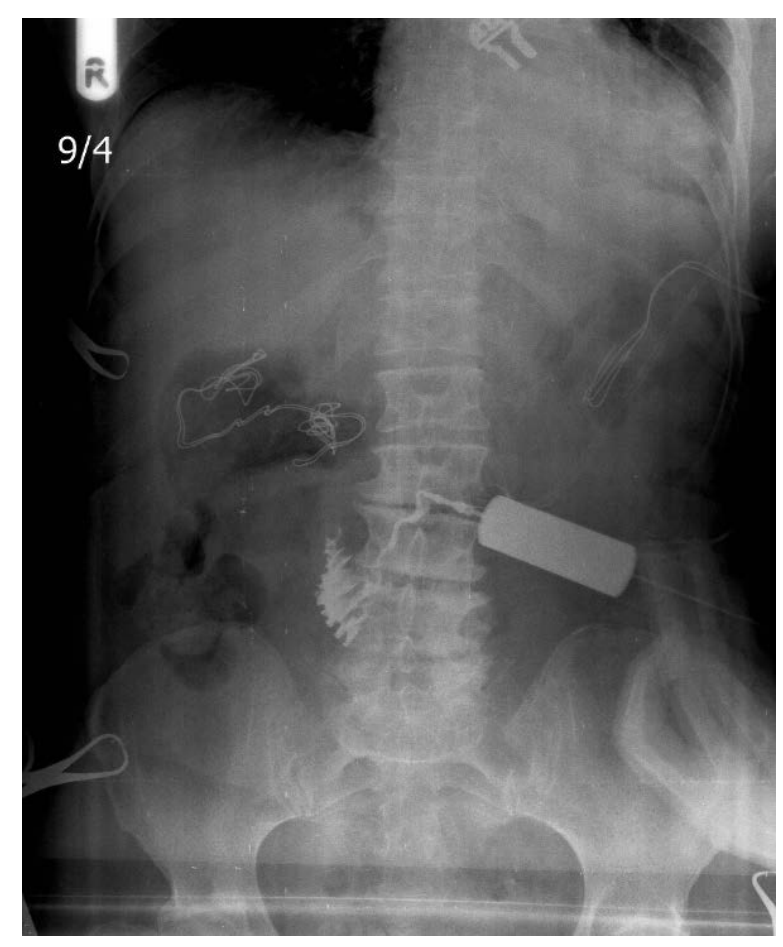

Figure 3. Cholangiography during surgery; confirmed the normal outflow to the duodenum and complete decompression of the common bile duct after cystic tumour resection.

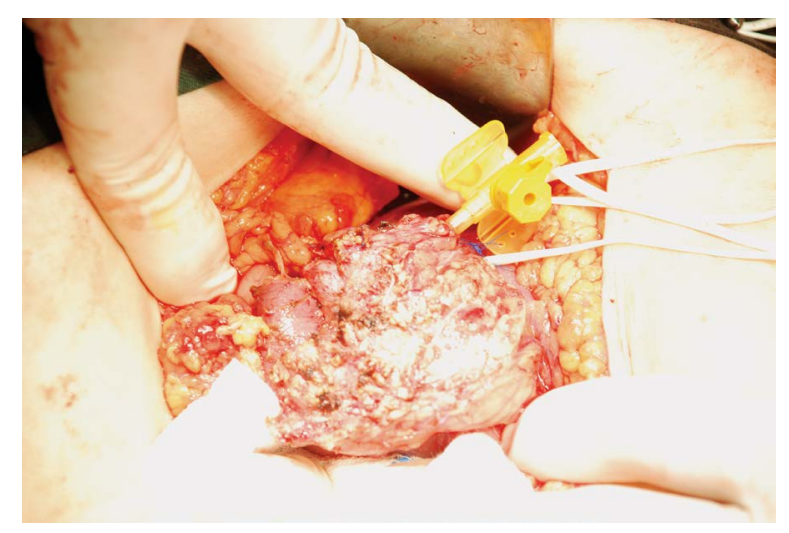

Figure 4. Cholangiography during surgery; confirmed the normal outflow to the duodenum and complete decompression of the common bile duct after cystic tumour resection.

drain was removed without signs of bile stasis. During the follow up the patient did not present symptoms of pancreatic exocrine insufficiency.

\section{Case 2}

A 57-year-old woman was admitted to the Department of General, Endocrine and Transplant Surgery due to symptomatic cholelithiasis. An abdominal CT was performed, and signs of pancreatic IPMN were found. The pancreas from the isthmus to the distal

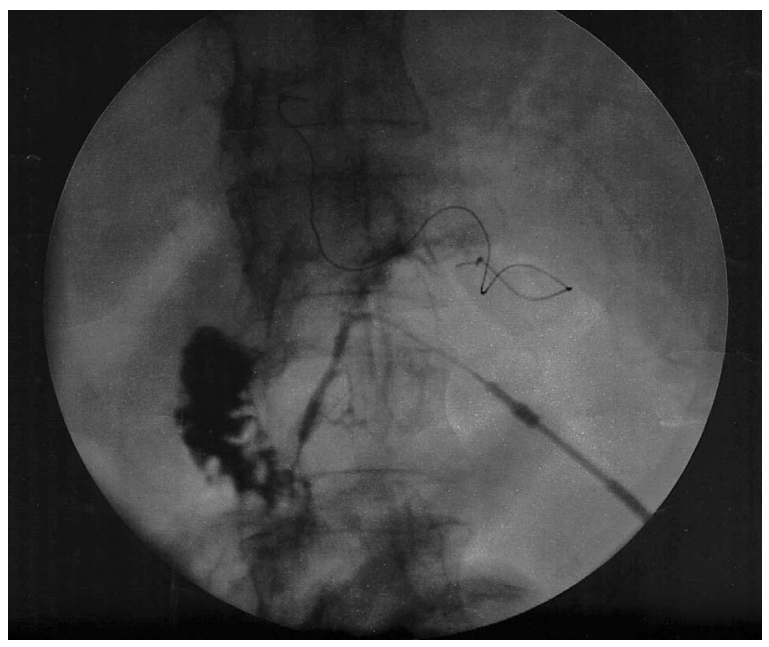

Figure 5. Pancreatography during surgery; showed a free flow to the duodenum without any pathology.

part of tail was atrophic, with visible massive distension of the Wirsung duct $(22 \mathrm{~mm})$ with secondary pancreatic duct dilatation. No unambiguous mass was found. The features of pancreatitis and fluid correlation in adherence were not shown. The image was interpreted first as an intraductal, papillary mucinous (pancreatic) neoplasm (IPMN), type 1, without involvement of the ventral bud duct. During admission the patient did not present any other symptoms. The patient was qualified for surgical treatment. The patient was an active woman with perfect physiological status and good biological state. After discussing all potential methods of treatment she requested pancreas sparing surgery, if possible, to increase her future life quality after pancreatic resection.

The cytology from the endoscopic retrograde cholangiopancreatography suggested a cystic mucosal lesion of low to medium dysplasia, an IPMN.

Surgery. During the operation an elastic mass was found covering the stem of the pancreas over an $8 \mathrm{~cm}$ length. On the anterior surface of the gland was found a clearly visible plane of fusion between prior embryological buds. Macroscopically, the cystic tumour did not reach this point. The Airid manoeuvre was performed and the splenic vessels were ligated. The pancreas was dissected and cut along the fusion plane. A normal size pancreatic duct was found. The pancreas and spleen were sent for intraoperative histopathological examination. A pancreatography was performed through the stump of the duct of the pancreatic remnant on the cut line, and showed a free flow to the duodenum without any pathology (Fig. 5). 
An intraoperative frozen section histopathological examination of the cut line revealed the presence of IPMN in the pancreatic duct. In this situation, instead of a partial pancreatic resection along the embryological fusion, a standard pylorus-preserving pancreaticoduodenectomy, also known as the Traverso-Longmire procedure, was performed.

The final histopathological examination revealed a colloid carcinoma in the body of the pancreas, pT3NOMO (Classification of Malignant Tumours $8^{\text {th }}$ Edition) with infiltration of fatty tissue. The patient underwent a second surgery 4 weeks later and a radicalisation was performed - resection of the retroperitoneal tissue. A histopathological examination showed no evidence of cancer infiltration or positive lymph nodes.

\section{DISCUSSION}

Pancreatic resection by a laparoscopic or open method is one of the most sophisticated procedures in abdominal surgery. It accompanies relatively large numbers of complications. The resection of the duodenum during pancreatic surgery determines the functional and endocrine status of the upper digestive tract. Duodenum sparing and pancreas sparing surgery are of major importance for patients concerning the post-operative quality of life $[4,14]$.

The embryonic development of the human pancreas comprises two embryo buds, and occurs about week 7 of pregnancy. The plane along the junction of the two parts is called the EFP $[3,8]$. The EFP is an anatomical feature that is important in planning pancreatic surgery. The differences between two embryo buds is described in pathology reports, and encourages surgeons to make the decision to operate along the $\operatorname{EFP}[3,8]$. The concept of EFP surgery is based on that of the embryo buds in the pancreas divisum. Anatomical cadaveric studies document that the EFP contains only the small pancreatic ducts and small vessels [8]. The concept was adapted to the normal pancreas after full fusion. Pancreatic bud sparing surgery can be performed with the dorsal and ventral bud. Several although not numerous papers confirm the feasibility of pancreatic partial resection along the $\operatorname{EFP}[1,3,4,8,14]$.

Surgical resection of pancreatic premalignant or for low-grade neoplasms might be also considered as gland sparing surgery $[4,5]$. Described elsewhere, the indications for pancreatic bud resection are IPMNs, neuroendocrine tumours, and pseudo-papillary tumours $[4,5,14]$. The pancreas is an organ without any defined parts or segments. The partial resection of the pancreas is based on enucleation of the tumours or segmental resections after gland transection. A pathology limited to pancreatic duct or pancreatic head cystic or neuroendocrine tumours might require a different kind of pancreas sparing surgery. Dorsal bud resection is attractive in upper aspect pancreatic head benign lesions, especially IPMN [15]. The clinical outcome or radicality of pancreatic sparing surgery is the same as large resections in patient series [15]. Indications for this kind of surgery are limited, and qualified to a more sophisticated option than the rule $[1,5,14]$. Although an experienced team was used and a high resolution CT was performed in case 2, a locally advanced cancer remained unrecognised. This demonstrates how difficult case selection can be.

The procedure for embryological bud resection is much more complicated, and related to a high rate of possible complications. On the other hand, high volume centres may offer this procedure at an acceptable rate of complications in selected cases.

\section{CONCLUSIONS}

In conclusion, an accurate diagnosis with a vascular anatomy and biliary and pancreatic duct configuration gave grounds to analyse the pancreas sparing surgery. The operation plan requires careful three-dimensional planning and an experienced team. Bipolar electrocautery, micro-surgical tools and intraoperative cholangiography and pancreatography are all helpful. The authors began the practical aspect of EFP preparation based on a cadaveric exercise before clinical implementation. During a dorsal primodium pancreatic resection it is possible to preserve the duodenum and distal bile duct. All arcades of the pancreatic head should be preserved to avoid ischaemia complications.

\section{REFERENCES}

1. Chunfu Z, Zhongzhi J, Xudong Z, et al. Anatomical segmentectomy of the pancreatic head along the embryological fusion plane: a case series and a literature review. Medicine. 2018; 18: e0623.

2. Iacono C, Ruzzenente A, Conci S, et al. Head dorsal pancreatectomy: an alternative to the pancreaticoduodenectomy for not enucleable benign or low-grade malignant lesions. Pancreatology. 2014; 14(5): 419-424, doi: 10.1016/j. pan.2014.07.014, indexed in Pubmed: 25163807.

3. Kimura W. Surgical anatomy of the pancreas for limited resection. J Hepatobiliary Pancreat Surg. 2000; 7(5): 473-479, doi: 10.1007/s005340070017, indexed in Pubmed: 11180873. 
4. Nakagohri T, Kinoshita T, Konishi M, et al. Inferior head resection of the pancreas for intraductal papillary mucinous neoplasms. J Hepatobiliary Pancreat Sci. 2010; 17(6): 798-802, doi: 10.1007/s00534-009-0173-8, indexed in Pubmed: 19727540.

5. Nishi T, Kawabata Y, Ishikawa N, et al. Intraductal papillary mucinous carcinoma of the pancreas associated with pancreas divisum: a case report and review of the literature. BMC Gastroenterol. 2015; 15: 78, doi: 10.1186/s12876015-0313-3, indexed in Pubmed: 26152300.

6. Ohwada S, Ogawa T, Kasahara M, et al. Ventral pancreas-preserving pancreatic head and body resection. Hepatogastroenterology. 2001; 48(42): 1622-1624, indexed in Pubmed: 11813586.

7. Ryu M, Takayama W, Watanabe K, et al. Ventral pancreatic resection for adenoma and low-grade malignancies of the head of the pancreas. Surg Today. 1996; 26(7): 476-481, doi: 10.1007/BF00311552, indexed in Pubmed: 8840427.

8. Sakamoto $Y$, Nagai $M$, Tanaka N, et al. Anatomical segmentectomy of the head of the pancreas along the embryological fusion plane: a feasible procedure? Surgery. 2000; 128(5): 822-831, doi: 10.1067/msy.2000.109611, indexed in Pubmed: 11056446.

9. Scatton O, Sauvanet A, Cazals-Hatem D, et al. Dorsal pancreatectomy: an embryology-based resection. J Gastrointest Surg. 2006; 10(3): 434-438, doi: 10.1016/j. gassur.2005.06.014, indexed in Pubmed: 16504892.
10. Skandalakis L, Rowe J, Gray S, et al. Surgical embryology and anatomy of the pancreas. Surg Clin North Am. 1993; 73(4): 661-697, doi: 10.1016/s0039-6109(16)46080-9.

11. Talbot ML, Foulis AK, Imrie CW. Total dorsal pancreatectomy for intraductal papillary mucinous neoplasm in a patient with pancreas divisum. Pancreatology. 2005; 5(2-3): 285-288, doi: 10.1159/000085284, indexed in Pubmed: 15855827.

12. Thayer SP, Fernández-del Castillo $\mathrm{C}$, Balcom JH, et al. Complete dorsal pancreatectomy with preservation of the ventral pancreas: a new surgical technique. Surgery. 2002; 131(5): 577-580, doi: 10.1067/msy.2002.123010, indexed in Pubmed: 12019413.

13. Uchida T, Takada T, Ammori BJ, et al. Three-dimensional reconstruction of the ventral and dorsal pancreas: a new insight into anatomy and embryonic development. J Hepatobiliary Pancreat Surg. 1999; 6(2): 176-180, doi: 10.1007/s005340050102, indexed in Pubmed: 10398906.

14. Wang X, Tan CL, Song HY, et al. Duodenum and ventral pancreas preserving subtotal pancreatectomy for low-grade malignant neoplasms of the pancreas: An alternative procedure to total pancreatectomy for low-grade pancreatic neoplasms. World J Gastroenterol. 2017; 23(35): 6457-6466, doi: 10.3748/wjg.v23.i35.6457, indexed in Pubmed: 29085195.

15. Yu-Wen $\mathrm{T}$, Rey-Heng Hu, Ji-Shiang $\mathrm{H}$, et al. Noninvasive pancreatic cystic neoplasms can be safely and effectively treated by limited pancreatectomy. Ann Surg Oncol. 2008; 15(1): 193-198, doi: 10.1245/s10434-007-9613-3, indexed in Pubmed: 17909909. 